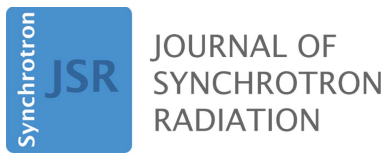

ISSN 1600-5775

Received 28 July 2021

Accepted 16 September 2021

Edited by Y. Amemiya, University of Tokyo, Japan

Keywords: free-electron laser; X-ray spectrometer; spectroscopy.

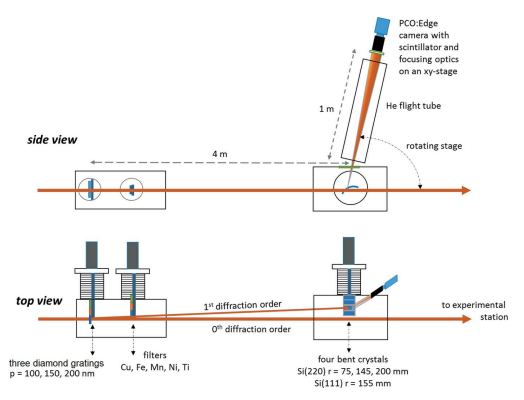

OPEN ๑ ACCESS

\section{Spectral monitoring at SwissFEL using a high-resolution on-line hard X-ray single-shot spectrometer}

\author{
Christian David, ${ }^{a *}$ Gediminas Seniutinas, ${ }^{\mathrm{a}}$ Mikako Makita, ${ }^{\mathrm{b}}$ Benedikt Rösner, ${ }^{\mathrm{a}}$ \\ Jens Rehanek, ${ }^{\mathrm{c}}$ Petri Karvinen, ${ }^{\mathrm{d}}$ Florian Löhl, ${ }^{\mathrm{a}}$ Rafael Abela, ${ }^{\mathrm{a}}$ Luc Patthey ${ }^{\mathrm{a}}$ and \\ Pavle Juranića
}

\footnotetext{
${ }^{\mathbf{a}}$ Paul Scherrer Institut, Forschungsstrasse 111, 5232 Villigen, Switzerland, ${ }^{\mathbf{b}}$ European XFEL GmbH, Holzkoppel 4 22869 Schenefeld, Germany, ${ }^{\mathbf{C}}$ Advanced Accelerator Technologies AG, 5234 Villigen, Switzerland, and

dinstitute of Photonics, University of Eastern Finland (UEF), Fl-80100 Joensuu, Finland.

*Correspondence e-mail: christian.david@psi.ch, christian.david@psi.ch
}

The performance and parameters of the online photon single-shot spectrometer (PSSS) at the Aramis beamline of the SwissFEL free-electron laser are presented. The device operates between the photon energies 4 and $13 \mathrm{keV}$ and uses diamond transmission gratings and bent Si crystals for spectral measurements on the first diffraction order of the beam. The device has an energy window of $0.7 \%$ of the median photon energy of the free-electron laser pulses and a spectral resolution (full width at half-maximum) $\Delta E / E$ on the order of $10^{-5}$. The device was characterized by comparing its performance with reference data from synchrotron sources, and a parametric study investigated other effects that could affect the reliability of the spectral information.

\section{Introduction}

The pulse-to-pulse measurement of spectra of self-amplified spontaneous emission (SASE) X-ray free-electron lasers (FELs) (Bergmann et al., 2017) is of fundamental importance for several experimental techniques ranging from resonant inelastic X-ray scattering (RIXS) (Lemke et al., 2013; Chergui, 2016; Obara et al., 2017; Park et al., 2019; Kimberg \& Rohringer, 2016; Błachucki et al., 2014; Kayser et al., 2019) to protein crystallography (Tono et al., 2015; Moreno-Chicano et al., 2019). The SASE process changes the spectral properties of the X-ray pulse on a pulse-to-pulse basis, which requires a device for online, non-invasive measurements of the X-ray spectra for both experimental spectral normalization and performance optimization (Rehanek et al., 2017). A good example of the use of an online spectrometer is in X-ray absorption spectroscopy (XAS), where measurements have been reported using both SASE and sample-transmitted spectra (Boutet \& Hunter, 2018; Katayama et al., 2013; Brenner et al., 2019). Such spectrometers are also useful in high-pressure or high-energy density science, where scans have to be performed with a small number of shots, preventing monochromator scans (Harmand et al., 2015). A large advantage in recording absorption spectra with the full SASE mode compared with the use of monochromatic light is that extreme intensity fluctuations are avoided, if the SASE spectrum does not contain the photon energy chosen by the monochromator (Boutet \& Hunter, 2018). The SASE spectrum also carries useful information to set up FEL operational parameters and develop new operating modes (Rehanek et al., 2017) and can be used to estimate pulse durations from the 
measurements of spectral spike widths (Malyzhenkov et al., 2020; Huang et al., 2017; Makita et al., 2015).

Several different spectrometer designs have been used at FELs for these kinds of spectral characterizations (Inubushi et al., 2012; Boesenberg et al., 2017; Rich et al., 2016; Svetina et al., 2016; Makita et al., 2015; Tono et al., 2013). Experiments at the Linac Coherent Light Source (LCLS) used bent $\mathrm{Si}$ crystals with transmission gratings (Makita et al., 2015). The bent Si crystals approach achieved high-resolution measurements (better than $0.2 \mathrm{eV}$ at $8.3 \mathrm{keV}$ ), but were limited in spectral range and lost about half of the photon flux due to poor transmission (Zhu et al., 2012) while the transmission gratings had a poorer resolution $(1.2 \mathrm{eV}$ at $6 \mathrm{keV}$ ) but much better transmission (Karvinen et al., 2012). The SPring-8 Angstrom Compact Free Electron Laser (SACLA) used a transmission grating in combination with an elliptical mirror and a flat $\mathrm{Si}$ crystal to deliver online spectra with tunability in both resolution and spectral range (Katayama et al., 2016). The Swiss-FEL photon single-shot spectrometer (PSSS) (Rehanek et al., 2017) combines a transmission grating with bent $\mathrm{Si}$ crystals to create a spectrometer that has a good resolution, large spectral range, and good transmission for online spectral measurements of photon energies between 4 and $13 \mathrm{keV}$. Using this setup, the first order of the diffracted beam is used for spectral or intensity monitoring, while the zeroth order is transmitted downstream of the experiments.

This work presents the achievements and the characterized capabilities of the PSSS as a single-shot online X-ray spectrometer. We demonstrate that the PSSS can deliver a full width at half-maximum $(\mathrm{FWHM})$ resolution of $\Delta E / E \simeq$ $5 \times 10^{-5}$ and a spectral window of up to $0.7 \%$ of the photon energy over the working range of the device.

\section{Setup}

The working principle of the PSSS is shown in Fig. 1. The diamond grating diffracts the incoming FEL beam in the horizontal plane, sending the first order to the bent crystal spectrometer while the zeroth order continues further downstream with $80 \%$ or more of the incoming flux. Monitoring the spectra online in this fashion reduces the heat load on the spectrometer optics (Boesenberg et al., 2017). The first order is Bragg-reflected from the bent Si crystal in the spectrometer and projected onto a detector, as shown in Fig. 1. The detector is a PCO:Edge 5.5 camera with an objective that is focused onto a Ce:YAG scintillator. The diffraction gratings have pitches of $100 \mathrm{~nm}, 150 \mathrm{~nm}$ and $200 \mathrm{~nm}$. The grating pitches are chosen such that the first-order diffracted beam is always far enough from the zeroth order so that the crystals can be put safely into it and do not block or affect the propagation of the main beam to the experimental station downstream. The first-order efficiency can be enhanced by tilting the diamond gratings up to $60^{\circ}$. For the bent $\mathrm{Si}$ crystals, three $\mathrm{Si}(220)$ crystals with bending radii of $75 \mathrm{~mm}, 145 \mathrm{~mm}$ and $200 \mathrm{~mm}$, and one $\mathrm{Si}(111)$ crystal with a bending radius of $155 \mathrm{~mm}$ can be chosen. All of the Si crystals are $10 \mu \mathrm{m}$ thick. More information about the PSSS construction are given by Rehanek et al. (2017) and Juranić et al. (2018).

Additional profile monitors situated both before and after the Bragg crystal chambers allow for the destructive observation of the diffracted beam and to see the Bragg diffraction in transmission. These monitors are Ce:YAG scintillators with a camera/lens unit (Juranić et al., 2018). Fig. 2(a) shows the diffracted beam on the profile monitor, while Fig. 2(b) shows
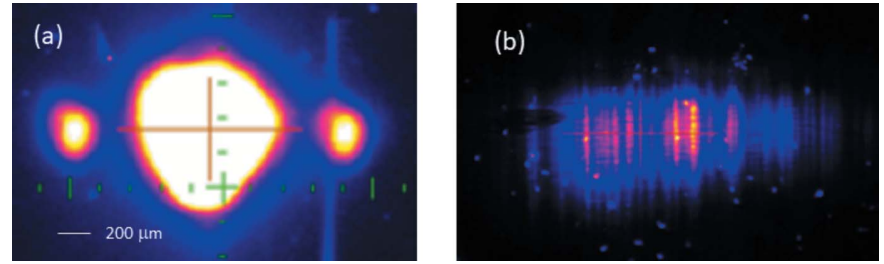

Figure 2

Diffracted orders of the full beam observed by the saturated profile monitors between the grating and Bragg crystal chambers $(a)$, and the raw image of the spectrum on the PSSS detector $(b)$. 

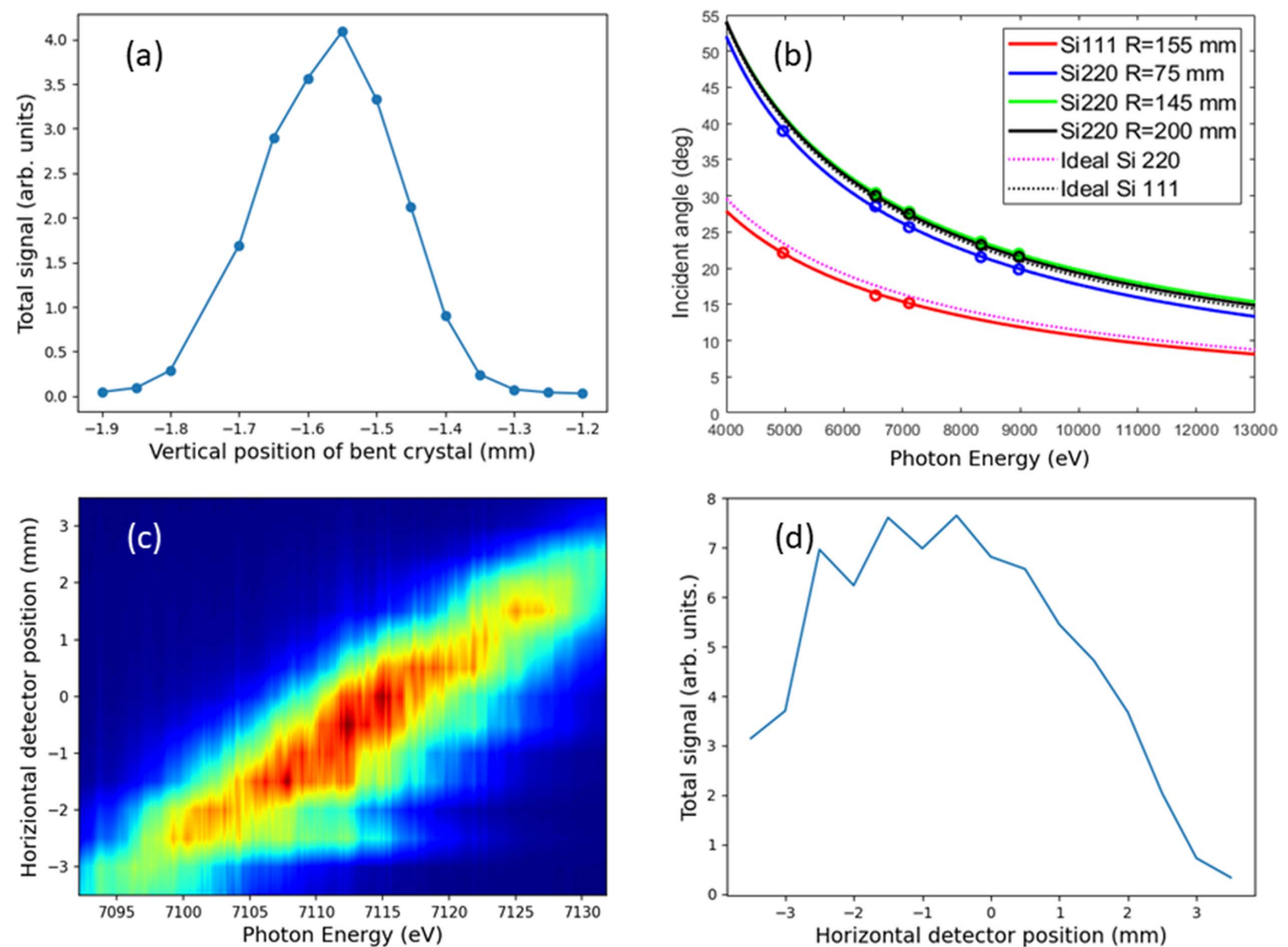

Figure 3

Vertical position scan for a $12 \mathrm{keV}$ beam of the Si bent crystals on the first-order diffraction $(a)$, the Bragg angles from various $K$-shell foil edges (circles) compared with ideal Bragg reflection curves for each crystal $(b)$, and the detector response tests as a function of detector position perpendicular to the Bragg reflection, showing the average spectra $(c)$ and integrated intensity and pulse energy $(d)$ at a photon energy of about $7.1 \mathrm{keV}$ and pulse energy of about $200 \mu \mathrm{J}$.

the raw image of the spectrum on the PCO.Edge camera of the spectrometer. The RMS size of the photon beam in the horizontal and vertical directions at the PSSS ranges from about 150 to $400 \mu \mathrm{m}$, depending on the photon energy and operating mode.

The PSSS setup requires precise alignment of the Si bent crystals to the beam. The vertical position of the crystal is the most important parameter to maximize the signal once the correct Bragg angles were selected for a chosen energy window. The setup is typically conducted in the non-invasive mode: the diamond gratings upstream diffract the beam, and the analyser crystals are placed in the first diffraction order. An exemplary measurement, conducted at $12 \mathrm{keV}$ photon energy and with a pulse energy of approximately $110 \mu \mathrm{J}$, is shown in Fig. 3(a).

The precise energy calibration of the spectrometer was performed by inserting several filter foils into the beam and identifying the transmission edges. By fitting the observed position on the camera to the expected Bragg angles, the geometry, miscut of the crystals and mounting tolerances were taken into account. The used foils were Ti $(K$-edge $4.966 \mathrm{keV}$, $10 \mu \mathrm{m}$ thickness), Mn ( $K$-edge $6.54 \mathrm{keV}, 20 \mu \mathrm{m}$ thickness), $\mathrm{Fe}$ ( $K$-edge $7.11 \mathrm{keV}, 10 \mu \mathrm{m}$ thickness), Ni $(K$-edge $8.33 \mathrm{keV}$, $12.5 \mu \mathrm{m}$ thickness) and $\mathrm{Cu}$ ( $K$-edge $8.98 \mathrm{keV}, 20 \mu \mathrm{m}$ thickness). The photon energy of the FEL was set to the absorption edges of the foils, and the Bragg angle of the PSSS was scanned to find the motor positions that matched the photon energies of the absorption edges for all crystals. The measured points were then used to fit a theoretical Bragg curve with an additional free parameter to take the miscut of the crystals into account. These fits were then used to create a look-up table for all relevant motor positions across the energy window for each crystal. The resulting points and Bragg curves are shown in Fig. 3(b). Note that we also show the Bragg curves without miscuts for $\mathrm{Si}(111)$ and $\mathrm{Si}(220)$ (dashed lines).

The sensitivity across the detector was investigated by scanning the detector position perpendicular to the Bragg reflection and comparing the integrated intensity against the simultaneous measurements taken with the online gas-based pulse energy monitor (Juranić et al., 2018). The field of view of the detector is about $4 \mathrm{~mm}$, so the spectra becomes cut off as one approaches that limit, and the integrated intensity drops off as a part of the spectrum is cut out, as shown in Figs. 3(c) and $3(d)$.

\section{PSSS performance and discussion}

\subsection{Operational parameters}

3.1.1. Si crystals and transmission gratings. The first step of the PSSS commissioning process was to determine the performance of different Si crystals and transmission gratings. From the Bragg angles [Fig. 3(b)], we can see the reachable energy windows for the different silicon crystals. The $\mathrm{Si}(220)$ 

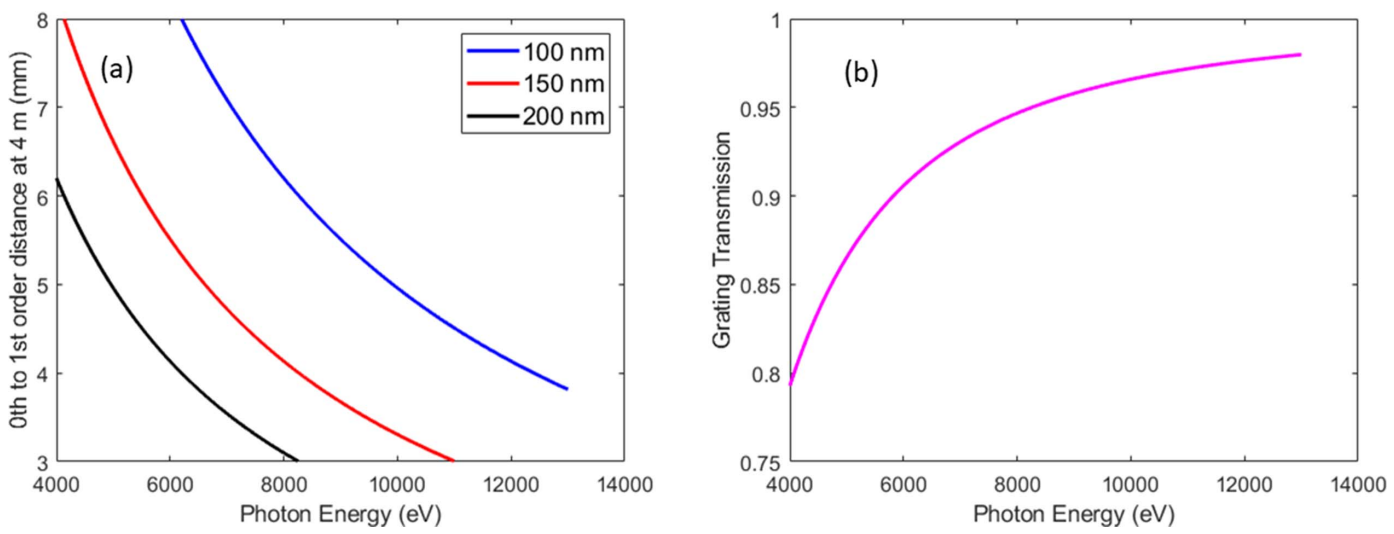

Figure 4

Separation from the zeroth- to first-order $(\mathrm{mm})$ beam at the bent crystals chamber from diffraction by different pitches of the diamond gratings $(a)$, zeroth-order transmission of the diamond gratings as a function of photon energy $(b)$.

reflection can be used over the complete range of the PSSS (4 to $13 \mathrm{keV}$ ), while the $\mathrm{Si}(111)$ reflections can be used from $4 \mathrm{keV}$ to $8 \mathrm{keV}$ due to the limited range of the detector rotation stage due to other beamline components, limiting us to Bragg reflections from $14^{\circ}$ to $60^{\circ}$.

Fig. 4(a) shows the dependence of the separation of the zeroth- and first-order beams as a function of photon energy for the three available diamond gratings with 100, 150 and $200 \mathrm{~nm}$ pitches. The region between $14^{\circ}$ and $60^{\circ}$ is where noninvasive operation of the PSSS is possible, corresponding to between $3 \mathrm{~mm}$ and $8 \mathrm{~mm}$ beam separation. Fig. 4(b) shows the calculated transmission of the diamond gratings as a function of photon energy, calculated with the Henke tables (Henke et al., 1993; http://henke.lbl.gov/optical_constants/tgrat2.html). The transmitted beam has a transmission between $80 \%$ at $4 \mathrm{keV}$ and $98 \%$ at $13 \mathrm{keV}$ photon energy. The transmission efficiency of the gratings has been measured and reported elsewhere (Juranic et al., 2019).

3.1.2. Si crystal alignment, energy calibration and detector sensitivity. The average integrated intensity of the spectra on the detector shows a very strong dependence on the vertical position of the bent $\mathrm{Si}$ crystal in the beam, as shown in Fig. 3(a). The integrated intensity drops by about $50 \%$ for a $110 \mu \mathrm{m}$ displacement of the crystal for an optimum position, highlighting the sensitivity of the device to misalignments and shifts in the beam position.

The difference between the measured Bragg angle values and those expected from the calculations are explained by the miscuts in the manufacturing process of the Si crystals (Rehanek et al., 2017). The offset between the measured crystal angles and the ideal Bragg angles is obvious in Fig. 3(b), where the three different $\operatorname{Si}(220)$ crystals have slightly different Bragg curves, offset from each other by a constant. The angular offsets of the Bragg angle for the four crystals were determined from these fits, and are shown in Table 1.

Fig. 3(c) shows the average spectra plotted as a function of the sensor position perpendicular to the Bragg reflection. The sensitivity seems to be homogeneous over the scanned region, though the spectra, and the integrated intensity, start being clipped and reduced as the spectrum is moved out of the
Table 1

Bragg angle offsets due to miscuts for the four bent crystals.

\begin{tabular}{lc}
\hline Crystal & Offset $\left(^{\circ}\right)$ \\
\hline $\mathrm{Si}(111), R=155 \mathrm{~mm}$ & -0.217 \\
$\mathrm{Si}(220), R=75 \mathrm{~mm}$ & -0.898 \\
$\mathrm{Si}(220), R=145 \mathrm{~mm}$ & 1.149 \\
$\mathrm{Si}(220), R=200 \mathrm{~mm}$ & 0.537 \\
\hline
\end{tabular}

sensor's field of view. Fig. 3(d) indicates that the best sensor position for the spectral center to minimize this clipping is between $-2.5 \mathrm{~mm}$ and $1 \mathrm{~mm}$, giving about a $3.5 \mathrm{~mm}$ effective field of view to reliably observe the spectrum.

3.1.3. FEL beam profile. Previous work has noted that the spectral intensity distribution can depend on the part of the FEL beam profile that is being Bragg reflected by the crystals (Makita et al., 2015; Rehanek et al., 2017). The homogeneity of the beam profile across the sampled portion of the beam being Bragg reflected should be as good as possible to ensure good spectral intensity measurements.

Fig. 5(a) shows the profile of the transmitted beam with a Bragg crystal positioned on the main beam (without the use of a diamond grating) that was acquired with a profile monitor downstream of the Bragg crystals (attenuated to avoid saturation). The diffracted portion of the beam is revealed by the small intensity drop in the beam center. The amount of the Bragg reflection is estimated as the maximum difference between the sides and the Bragg dip in the middle of the profile, as shown in Fig. 5(b). The dip in signal due to the Bragg reflection is about $5 \%$ near the maximum. From the tiny amount of the diffraction and the uniformity of the transmitted beam profile, we anticipate that the influence of the intensity on the measured spectral distribution of the beam is negligible as long as the Bragg crystal is centered.

3.1.4. Measured energy resolution and spectral range. Fig. 6 shows the expected PSSS resolution at several different photon energies. The measurements were taken with the PSSS non-invasive mode, looking at the first order of the transmission grating. There are two measures to determine the resolution of a spectrometer: the minimum spike width that 

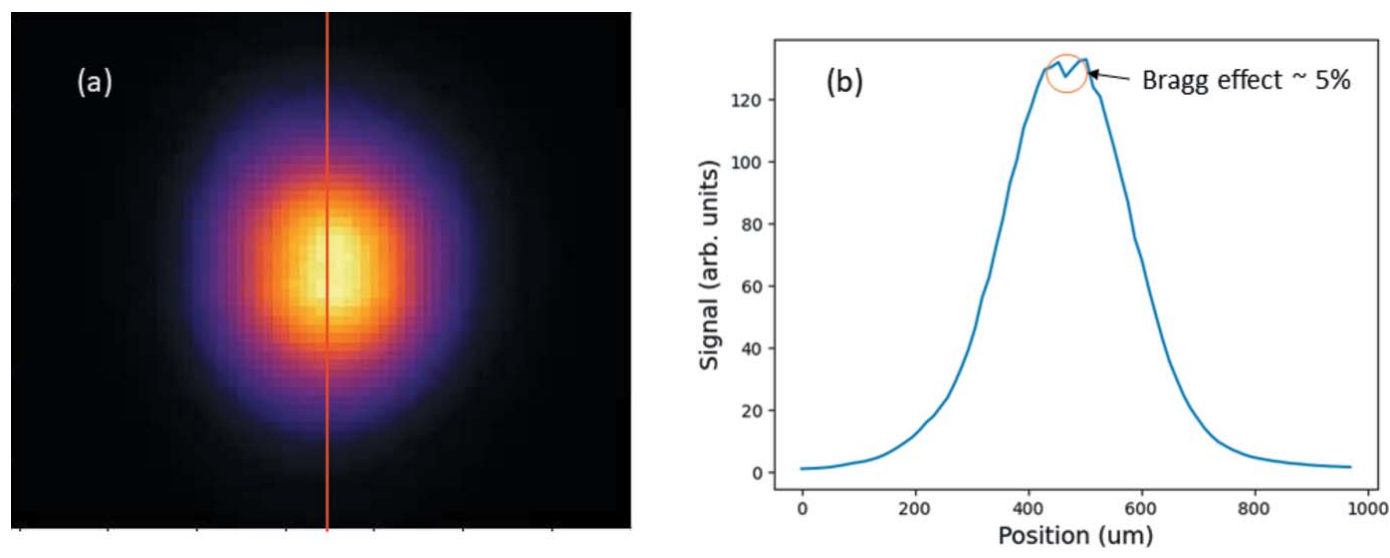

Figure 5

(a) Color map of the FEL beam profile when the bent crystals are in the zeroth-order beam and attenuated to prevent saturation effects. (b) The projection of the pixels along the red line in (a) that shows the loss of signal due to the Bragg reflection in transmission, accounting for about $5 \%$ of the intensity at the maximum.
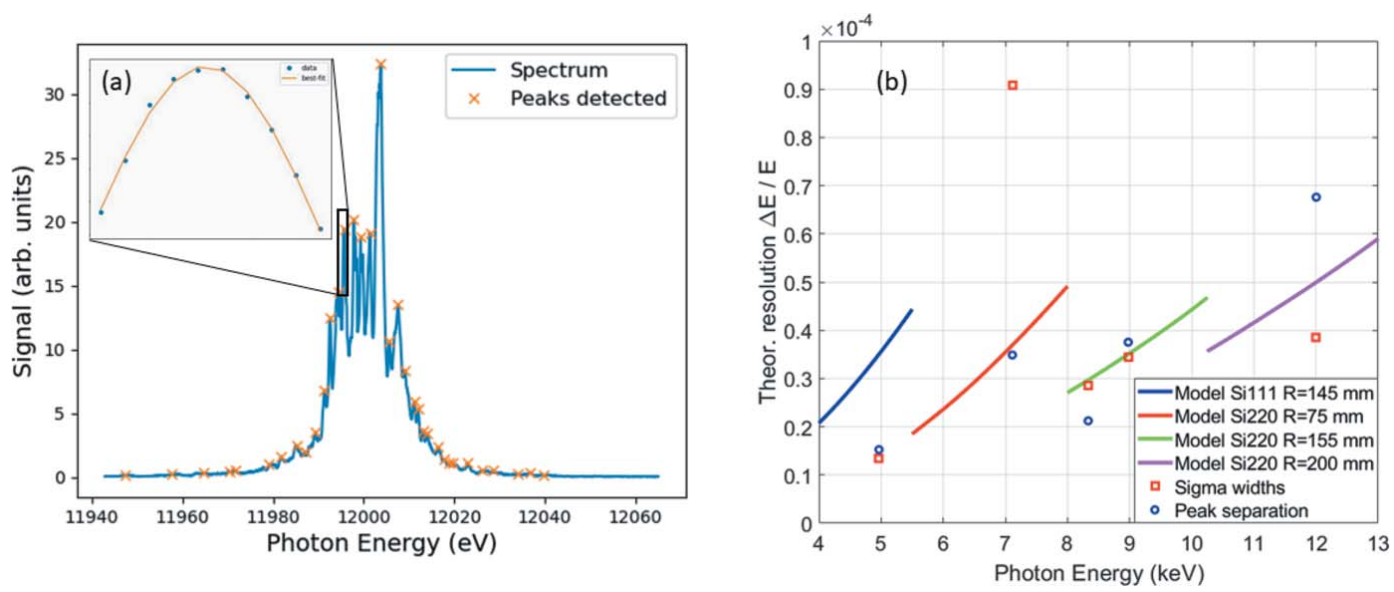

Figure 6

(a) A spectrum used for peak finding and Gaussian curve fitting taken at $12 \mathrm{keV}$. (b) Resolution measurements of the PSSS at the filter foil $K$-edges and at $12 \mathrm{keV}$ compared with theoretical expectations.

it can resolve, and the minimum peak separation that is still visible. The evaluation for these two values was performed using the Python Scipy library which evaluated the peaks whose prominence was at least ten times above the standard deviation of the noise in the background signal at the edges of the spectrum. The smallest separation between these peaks was considered as the best resolution observed. The peaks were then fitted with a Gaussian model (including an offset) to find their widths. Those peaks that had the offset near the background level of the spectrum were compared, and the smallest sigma values of these peaks was taken as the best sigma width resolution. A thousand spectra were evaluated to find the smallest sigma values and peak separations for each photon energy. Fig. 6 shows the minimum spike width and peak separation that were observed at $4.966 \mathrm{keV}, 7.114 \mathrm{keV}$, $8.333 \mathrm{keV}, 8.996 \mathrm{keV}$ and $12.015 \mathrm{keV}$ photon energies and compares them with the expected values. They are also compared with each other and expected theoretical values in Table 2, which were evaluated using the expected beam size for that photon energy.

Note that the measurements for the minimum spike width are a convolution of the resolution of the spectrometer and
Table 2

Expected theoretical and measured resolutions by photon energy; the measured resolutions were evaluated using peak separations and by looking at the smallest peak widths found.

\begin{tabular}{llll}
\hline $\begin{array}{l}\text { Photon } \\
\text { energy }(\mathrm{eV})\end{array}$ & $\begin{array}{l}\text { Expected } \\
\text { resolution }(\mathrm{eV})\end{array}$ & $\begin{array}{l}\text { Peak } \\
\text { separation }(\mathrm{eV})\end{array}$ & $\begin{array}{l}\text { Sigma } \\
\text { width }(\mathrm{eV})\end{array}$ \\
\hline 4966 & 0.174 & 0.076 & 0.066 \\
7112 & 0.263 & 0.248 & 0.646 \\
8333 & 0.250 & 0.177 & 0.237 \\
8996 & 0.315 & 0.337 & 0.309 \\
12015 & 0.610 & 0.816 & 0.462 \\
\hline
\end{tabular}

the natural spike width of the FEL spectrum, which likely limits the measurable energy resolution.

The sigma and peak separation measurements are specifically close to the expected energy resolution for the $\mathrm{Si}(220)$ crystal with $R=155 \mathrm{~mm}$, and reasonably close to the crystal with $R=200 \mathrm{~mm}$. It is possible that the photon spectrum at this energy was not as optimized as the other ones, causing the spectrum itself to have broader spikes. However, the resolution of the spectrometer stays in the $10^{-5} \Delta E / E$ resolution range for all measurements, matching the expectations set during the device design. 


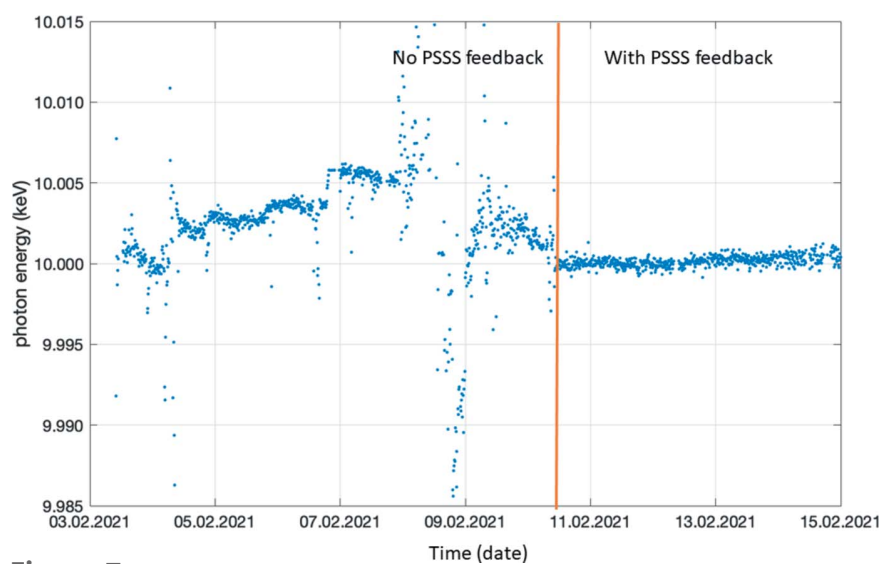

Figure 7

The spectral center of mass evaluated from the PSSS before (left of orange line) and after (right of orange line) the PSSS photon energy feedback was enabled on the machine during a week of operation.

The spectral range of the PSSS matched the theoretical estimates (Rehanek et al., 2017), ranging between $0.56 \%$ and $0.74 \%$ with the correct selection of Bragg crystals and bending radii. $\mathrm{Si}(220)$ crystals with bending radii of $200 \mathrm{~mm}, 145 \mathrm{~mm}$ and $75 \mathrm{~mm}$ are used for ranges of $5.5-8 \mathrm{keV}, 8-10.5 \mathrm{keV}$ and 10.5-13 keV, respectively. A $\mathrm{Si}(111)$ crystal with a bending radius of $155 \mathrm{~mm}$ is used to provide a similar spectral range for photon energies between 4 and $5.5 \mathrm{keV}$. The range is large enough to clearly visualize the standard mode spectrum and its tails, as seen in Fig. 6.

\subsection{Experience with the PSSS}

The PSSS went into full operation for users and machine operators after the implementation of a user-friendly graphical user interface for spectral measurements based on look-up tables created from the calibration data. To further help with the optimization of the FEL spectral settings, a fast algorithm was developed to evaluate the center of energy and the FWHM of a Gaussian fit to the smoothed spectrum on a shot-to-shot basis. Further tools created by the accelerator scientists use this data for feedbacks for the machine, and the PSSS is being used regularly to correct drifts and instabilities of the mean photon energy and spectral width via a feedback system and an optimizer to the SwissFEL accelerator settings. The PSSS has been used even when the end-stations use monochromators for an experiment, as the PSSS spectral bandwidth evaluation can be used to optimize the smallest bandwidth the FEL can achieve so that the maximum amount of photons possible pass through a monochromator's bandpass, increasing the signal at the experiment. A good example of such an optimization feedback, and its effect on stabilizing the photon energy, is shown in Fig. 7. Overall, the device has been extremely helpful for machine optimization and experimental work.

\section{Conclusions}

The PSSS enables the online monitoring of shot-to-shot fluctuations in spectral properties of the FEL, delivering a spectral range of up to $0.76 \%$ of the photon energy, and a resolution of bandwidths $\Delta E / E<5 \times 10^{-5}$, consistent with the theoretical estimates. Using $\mathrm{Fe}, \mathrm{Ni}$ and $\mathrm{Cu} K$-edges from XAS spectra from reference data from synchrotrons, the spectrometer was calibrated to be capable of visualizing the FEL spectrum in the standard mode while preserving high-resolution visualization of individual spikes. The PSSS enables the operators and users of the Aramis beamline to monitor and optimize the performance of the FEL and the experiment online, and is a crucial component of the SwissFEL diagnostics suite.

\section{Acknowledgements}

The authors would like to thank the SwissFEL operation team, especially Nicole Hiller, Sven Reiche, Didier Voulot, Thomas Schietinger, Thomas Marder, Peter Hottinger and Martin Brinkmann as well as the scientific staff of the Alvra end-station for their support, especially Christopher Milne and Claudio Cirelli. Likewise the authors would like to acknowledge the support from the controls team, especially from Thierry Zamofing and the useful discussions with Christopher Arrell.

\section{Funding information}

The following funding is acknowledged: H2020 European Research Council [grant No. 654360 NFFA-Europe (BR)].

\section{References}

Bergmann, U., Yachandra, V. K. \& Yano, J. (2017). X-ray Free Electron Lasers: Applications in Materials, Chemistry and Biology. Royal Society of Chemistry.

Błachucki, W., Szlachetko, J., Hoszowska, J., Dousse, J. C., Kayser, Y., Nachtegaal, M. \& Sá, J. (2014). Phys. Rev. Lett. 112, 173003.

Boesenberg, U., Samoylova, L., Roth, T., Zhu, D. L., Terentyev, S., Vannoni, M., Feng, Y. P., van Driel, T. B., Song, S., Blank, V., Sinn, H., Robert, A. \& Madsen, A. (2017). Opt. Express, 25, 2852-2862.

Boutet, S. F. P. \& Hunter, M. S. (2018). X-ray Free Electron Lasers: A Revolution in Structural Biology. Springer.

Brenner, G., Dziarzhytski, S., Miedema, P. S., Rösner, B., David, C. \& Beye, M. (2019). Opt. Lett. 44, 2157-2160.

Chergui, M. (2016). Struct. Dyn. 3, 031001.

Harmand, M., Ravasio, A., Mazevet, S., Bouchet, J., Denoeud, A., Dorchies, F., Feng, Y., Fourment, C., Galtier, E., Gaudin, J., Guyot, F., Kodama, R., Koenig, M., Lee, H. J., Miyanishi, K., Morard, G., Musella, R., Nagler, B., Nakatsutsumi, M., Ozaki, N., Recoules, V., Toleikis, S., Vinci, T., Zastrau, U., Zhu, D. \& Benuzzi-Mounaix, A. (2015). Phys. Rev. B, 92, 024108.

Henke, B. L., Gullikson, E. M. \& Davis, J. C. (1993). Atom. Data Nucl. Data Tables, 55, 349.

Huang, S., Ding, Y., Feng, Y., Hemsing, E., Huang, Z., Krzywinski, J., Lutman, A. A., Marinelli, A., Maxwell, T. J. \& Zhu, D. (2017). Phys. Rev. Lett. 119, 154801.

Inubushi, Y., Tono, K., Togashi, T., Sato, T., Hatsui, T., Kameshima, T., Togawa, K., Hara, T., Tanaka, T., Tanaka, H., Ishikawa, T. \& Yabashi, M. (2012). Phys. Rev. Lett. 109, 144801.

Juranić, P., Rehanek, J., Arrell, C. A., Pradervand, C., Ischebeck, R., Erny, C., Heimgartner, P., Gorgisyan, I., Thominet, V., Tiedtke, K., Sorokin, A., Follath, R., Makita, M., Seniutinas, G., David, C., Milne, C. J., Lemke, H., Radovic, M., Hauri, C. P. \& Patthey, L. (2018). J. Synchrotron Rad. 25, 1238-1248.

Juranic, P., Tiedtke, K., Owada, S., Tanaka, T., Jastrow, U., Sorokin, A., Patthey, L., Mankowsky, R., Degenhardt, M., Arbelo, Y., Arrell, 
C., Smedley, J., Bohon, J. \& Follath, R. (2019). J. Synchrotron Rad. 26, 2081-2085.

Karvinen, P., Rutishauser, S., Mozzanica, A., Greiffenberg, D., Juranić, P. N., Menzel, A., Lutman, A., Krzywinski, J., Fritz, D. M., Lemke, H. T., Cammarata, M. \& David, C. (2012). Opt. Lett. 37, 5073-5075.

Katayama, T., Inubushi, Y., Obara, Y., Sato, T., Togashi, T., Tono, K., Hatsui, T., Kameshima, T., Bhattacharya, A., Ogi, Y., Kurahashi, N., Misawa, K., Suzuki, T. \& Yabashi, M. (2013). Appl. Phys. Lett. 103, 131105.

Katayama, T., Owada, S., Togashi, T., Ogawa, K., Karvinen, P., Vartiainen, I., Eronen, A., David, C., Sato, T., Nakajima, K., Joti, Y., Yumoto, H., Ohashi, H. \& Yabashi, M. (2016). Struct. Dyn. 3, 034301.

Kayser, Y., Milne, C., Juranić, P., Sala, L., Czapla-Masztafiak, J., Follath, R., Kavčič, M., Knopp, G., Rehanek, J., Błachucki, W., Delcey, M. G., Lundberg, M., Tyrała, K., Zhu, D. L., Alonso-Mori, R., Abela, R., Sá, J. \& Szlachetko, J. (2019). Nat. Commun. 10, 4761.

Kimberg, V. \& Rohringer, N. (2016). Struct. Dyn. 3, 034101.

Lemke, H. T., Bressler, C., Chen, L. X., Fritz, D. M., Gaffney, K. J., Galler, A., Gawelda, W., Haldrup, K., Hartsock, R. W., Ihee, H., Kim, J., Kim, K. H., Lee, J. H., Nielsen, M. M., Stickrath, A. B., Zhang, W. K., Zhu, D. L. \& Cammarata, M. (2013). J. Phys. Chem. A, 117, 735-740.

Makita, M., Karvinen, P., Zhu, D., Juranic, P. N., Grünert, J., Cartier, S., Jungmann-Smith, J. H., Lemke, H. T., Mozzanica, A., Nelson, S., Patthey, L., Sikorski, M., Song, S., Feng, Y. \& David, C. (2015). Optica, 2, 912-916.

Malyzhenkov, A., Arbelo, Y. P., Craievich, P., Dijkstal, P., Ferrari, E., Reiche, S., Schietinger, T., Juranić, P. \& Prat, E. (2020). Phys. Rev. Res. 2, 042018 .
Moreno-Chicano, T., Ebrahim, A., Axford, D., Appleby, M. V., Beale, J. H., Chaplin, A. K., Duyvesteyn, H. M. E., Ghiladi, R. A., Owada, S., Sherrell, D. A., Strange, R. W., Sugimoto, H., Tono, K., Worrall, J. A. R., Owen, R. L. \& Hough, M. A. (2019). IUCrJ, 6, 1074 1085.

Obara, Y., Ito, H., Ito, T., Kurahashi, N., Thürmer, S., Tanaka, H., Katayama, T., Togashi, T., Owada, S., Yamamoto, Y.-I., Karashima, S., Nishitani, J., Yabashi, M., Suzuki, T. \& Misawa, K. (2017). Struct. Dyn. 4, 044033.

Park, S. H., Yoon, J., Kim, C., Hwang, C., Kim, D.-H., Lee, S.-H. \& Kwon, S. (2019). J. Synchrotron Rad. 26, 1031-1036.

Rehanek, J., Makita, M., Wiegand, P., Heimgartner, P., Pradervand, C., Seniutinas, G., Flechsig, U., Thominet, V., Schneider, C. W., Fernandez, A. R., David, C., Patthey, L. \& Juranić, P. (2017). J. Instrum. 12, P05024.

Rich, D., Zhu, D., Turner, J., Zhang, D., Hill, B. \& Feng, Y. (2016). J. Synchrotron Rad. 23, 3-9.

Svetina, C., Cocco, D., Mahne, N., Raimondi, L., Ferrari, E. \& Zangrando, M. (2016). J. Synchrotron Rad. 23, 35-42.

Tono, K., Nango, E., Sugahara, M., Song, C., Park, J., Tanaka, T., Tanaka, R., Joti, Y., Kameshima, T., Ono, S., Hatsui, T., Mizohata, E., Suzuki, M., Shimamura, T., Tanaka, Y., Iwata, S. \& Yabashi, M. (2015). J. Synchrotron Rad. 22, 532-537.

Tono, K., Togashi, T., Inubushi, Y., Sato, T., Katayama, T., Ogawa, K., Ohashi, H., Kimura, H., Takahashi, S., Takeshita, K., Tomizawa, H., Goto, S., Ishikawa, T. \& Yabashi, M. (2013). New J. Phys. 15, 083035 .

Zhu, D. L., Cammarata, M., Feldkamp, J. M., Fritz, D. M., Hastings, J. B., Lee, S., Lemke, H. T., Robert, A., Turner, J. L. \& Feng, Y. P. (2012). Appl. Phys. Lett. 101, 034103. 